DOI: https://doi.org/10.11144/Javeriana.upsy16-3.pcmd

\title{
El papel de la comunicación, la motivación y el disfrute sobre el compromiso en practicantes de musculación ${ }^{*}$
}

\section{The Role of Communication, Motivation and Enjoyment over Commitment in Fitness}

\author{
FERnANDA Borges-Silva ${ }^{\mathrm{a}}$ \\ Universidad Católica San Antonio de Murcia, España \\ ORCID: http://orcid.org/0000-0002-7429-9290 \\ Fernando Navarro López \\ Universidad Católica San Antonio de Murcia, España \\ Elisa HuÉsCAR \\ Universidad Miguel Hernández de Elche, España \\ Juan Antonio Moreno-Murcia \\ Universidad Miguel Hernández de Elche, España
}

\footnotetext{
a Autor de correspondencia. Correo electrónico: bsfernanda@ucam.edu

Para citar este artículo: Borges-Silva, F., Navarro López, F., Huéscar, E., \& Moreno-Murcia, J. A. (2017). El papel de la comunicación, la motivación y el disfrute sobre el compromiso en practicantes de musculación. Universitas Psychologica, 16(3), 1-9. https://doi.org/ 10.11144/Javeriana.upsy16-3.pcmd
}

\section{RESUMEN}

El objetivo de este estudio fue comprobar la predicción del compromiso en practicantes de musculación a través de las estrategias de comunicación (verbal, no verbal y evitación de barreras) del monitor, la motivación intrínseca y el disfrute en la actividad. Participaron 287 personas practicantes de musculación de centros deportivos, a los que se les midió la percepción de comunicación y las barreras de comunicación con el técnico, la motivación intrínseca, el disfrute y el grado de compromiso deportivo. El resultado del análisis de regresión lineal mostró que el uso adecuado de estrategias de comunicación verbal, no verbal y la evitación de barreras en la comunicación, así como altos niveles de motivación intrínseca y de disfrute en la práctica predecían el compromiso futuro de los practicantes. De esta forma, el uso de estrategias eficaces de comunicación del técnico de musculación durante la interacción con los practicantes, siempre que la motivación sea alta, posibilitaría una mayor adherencia a la práctica.

Palabras clave

comunicación; motivación intrínseca; disfrute; musculación.

\begin{abstract}
The purpose of this paper was to test the predictive power of the channels of communication used by fitness monitor, the intrinsic motivation and the enjoyment of the activity, in the commitment of fitness. 287 gym fitness were involved in the study to which were analyzed the degree of perceived communication and the communicational barriers with the monitor, the intrinsic motivation, the enjoyment and the level of sport commitment. The result of linear regression analysis showed that the use of channels of communication verbal, no-verbal and the avoidance of communication barriers, as well as high levels of intrinsic motivation and enjoyment during the practice, predicted a future commitment of the
\end{abstract}


participants. In this way, the use of effective communication strategies by the fitness monitor during the interaction with practitioners would increase the motivation of individuals allowing a greater adherence to practice.

Keywords

communication; intrinsic motivation; enjoyment; fitness.

En los últimos años, la práctica de actividad física ha cobrado un papel fundamental en la vida de las personas. Se ha producido un incremento de su demanda social debido a la nueva conciencia generalizada por la salud y el cuidado del cuerpo (Consejo Superior de Deporte [CSD], 2010). Así pues, en la encuesta de hábitos deportivos de los españoles, entre los principales motivos de práctica para la realización de ejercicio destacan la diversión y la mejora de la salud (García Ferrando, 2006). Por tanto, sabiendo de los numerosos beneficios del ejercicio físico a nivel psicológico, físico y social, sería primordial tener en cuenta los motivos de práctica, ya que su naturaleza podría revelar algunas claves sobre el compromiso deportivo de la persona. Un estudio realizado con practicantes de entrenamiento con pesas en gimnasio apuntó que el motivo principal para iniciarse en esta actividad varía entre hombres y mujeres jóvenes. Así pues, ellas inician la actividad para controlar el peso y ellos para mantener la forma física (Arbinaga \& García, 2003).

Además, paralelamente, también ha aumentado el interés tanto de las instituciones como de los profesionales por mejorar los principales componentes del proceso de enseñanza-aprendizaje durante la actividad física (Muñoz, Carreras, \& Braza, 2004; Tobón, 2004). La mayor parte del esfuerzo que emplea el profesional es utilizada para tomar decisiones sobre qué aspecto comunicar para conseguir los objetivos propuestos. La comunicación se considera un proceso que tiene lugar durante la interacción social, de carácter verbal o no verbal, en la que existe la intención de transmitir un mensaje que puede influir, con y sin intención, en el comportamiento de las personas que se encuentran en dicho proceso (Hernández \& Garay, 2003).

En esta línea, algunos estudios acerca de la comunicación han destacado el papel de las habilidades comunicativas sobre una serie de variables como el éxito personal (Morreale, Osborn, \& Pearson, 2000), la satisfacción (Anderson \& Martin, 1995), la cohesión (Smaby, Harrison, \& Nelson, 1995) y la motivación (Moreno-Murcia, Huéscar, Peco, Alarcón, \& Cervelló, 2013). En el ámbito de la educación física (Amorose \& Smith, 2003; Koka \& Hein, 2005) y el deporte (Sánchez \& Viciana, 2002), algunos trabajos destacan el papel del feed-back (esto es, la información sobre las ejecuciones de los participantes) por su vinculación con el dominio de una actividad y el esfuerzo, señalando además la recomendación de aplicar feed-back de tipo positivo y sobre el conocimiento del rendimiento. Además, junto a los aspectos verbales, el canal de comunicación no verbal constituye un conducto de información continua percibida por los practicantes, cuya interpretación puede provocar una influencia directa sobre la motivación de la persona.

Según Poyatos (2003), en el proceso de comunicación, el mensaje verbal en sí prácticamente no tiene importancia para el interlocutor, recayendo sobre los aspectos no verbales el mayor peso de la interpretación del discurso. En este sentido, resulta fundamental minimizar todos aquellos aspectos de la comunicación que pudieran suponer un obstáculo para que el mensaje entre el emisorreceptor no solo fluya adecuadamente, sino que además contribuya a que la persona se sienta escuchada y valorada. En este sentido, se convierten en barreras de comunicación, por ejemplo, interrumpir a una persona mientras habla o no empatizar. Recientemente, el papel de la evitación de las barreras de la comunicación sobre la motivación intrínseca de los participantes viene siendo recomendado en la literatura científica, apostando por la creación de climas que estimulen a través de una comunicación multicanal (donde participen elementos verbales y no verbales) la oportunidad de expresión libre de ideas, de elección de 
tareas que inspiren seguridad y que estimulen la mejora personal (Moreno, González-Cutre, Martín-Albo, \& Cervelló, 2010; MorenoMurcia et al., 2013). En este sentido, trabajos recientes (Reeve et al., 2014) enfocados en los estilos interpersonales del técnico/docente señalan que la utilización de estilos que apoyen a la autonomía (minimizando la presión y animando hacia la acción) del practicante/ discente frente a estilos controladores (donde se impone la forma de proceder del técnico/ docente) está relacionado positivamente con la motivación autodeterminada (Haerens, Aelterman, Vansteenkiste, Soenens, \& Van Petegem, 2015).

Por otra parte, considerando que un escenario de práctica estimulante donde primen los aspectos relacionados con el disfrute, esto es, un estado positivo de afecto en el que se dan sentimientos como el gusto, la diversión y el placer (Scanlan \& Simons, 1992) sobre la competición, podría relacionarse con una mayor motivación intrínseca; algunos estudios han comprobado relaciones positivas y significativas entre ambas variables (Moreno, González-Cutre, Martínez, Alonso, \& López, 2008). Además, en diferentes investigaciones se ha mostrado que el disfrute en la actividad física se sitúa como uno de los motivos principales de la población para dicha práctica (Castillo \& Balaguer, 2001). Por tanto, existe una relación positiva del disfrute con la motivación, y negativa con la desmotivación, lo que indica que a mayor disfrute mayor motivación, y viceversa.

Por último, Sousa, Torregrosa, Viladrich, Villamarín y Cruz (2007) relacionaron el compromiso deportivo como un fuerte predictor del abandono deportivo, afirmando que cuanto mayor es el compromiso deportivo la posibilidad de abandono en el deporte es menor. Además, estos autores también afirman que el entrenador juega un papel importante en la diversión de los deportistas y en el compromiso de estos. Así pues, generar en la práctica de actividad física un clima que tenga en cuenta al practicante, permitiendo que participe en el proceso de toma de decisiones valorando y atendiendo su opinión, podría ser determinante en su compromiso deportivo futuro. Es el entrenador/monitor el responsable de concebir este clima, de forma que el deportista pueda participar en la actividad a lo largo del tiempo (Moreno, Cervelló, Marcos, \& Martín, 2010).

Por este motivo, el objetivo de este estudio fue comprobar la predicción del compromiso de los practicantes de musculación a través de las estrategias de comunicación (verbal, no verbal y evitación de barreras) del monitor, la motivación intrínseca y el disfrute en la actividad. Se espera que la comunicación prediga positivamente el compromiso deportivo futuro.

\section{Método}

\section{Participantes}

La muestra del estudio estuvo compuesta por 287 practicantes. Un $54.4 \%(n=156)$ eran hombres y un $45.6 \%(n=131)$ mujeres que realizaban entrenamiento de musculación de forma no competitiva en 12 centros deportivos de una gran ciudad española. Las edades de los participantes oscilaban entre los 18 y 35 años $(M=25.12, D E=4.93)$. El tiempo que los participantes llevaban realizando musculación era de un año, aproximadamente. Para escoger la muestra, se llevó a cabo una selección de centros atendiendo a un muestreo aleatorio por conglomerados (Azorín \& Sánchez-Crespo, 1986).

\section{Medidas}

\section{Comunicación verbal y no verbal}

Se usó la escala creada por Moreno-Murcia et al. (2012), diseñada para medir la comunicación verbal y no verbal que el técnico muestra a la hora de transmitir información al practicante. El cuestionario, encabezado por el enunciado "El monitor/a, en la sala de fitness, cuando me surge cualquier duda/imprevisto..." se compone de un total de siete ítems, cuatro para el factor Comunicación Verbal (p. ej., "Se explica de 
forma breve y concisa") y tres para el factor de Comunicación No Verbal (p. ej., "Cuando se dirige a mí, refuerza la comunicación con gestos, miradas, etc."). Las respuestas fueron puntuadas en una escala tipo Likert de 1 (Totalmente en desacuerdo) a 5 (Totalmente de acuerdo). La consistencia interna fue de 0.7 para la comunicación verbal y de 0.7 para la comunicación no verbal.

\section{Barreras de comunicación}

Se usó la escala creada por Moreno-Murcia et al. (2012) construida para medir la creación de un clima que minimizara las barreras de comunicación. El cuestionario, encabezado por el enunciado: "Durante mi sesión, el monitor/ entrenador de la sala de musculación..." se compone de nueve ítems (p. ej., "No me interrumpe cuando hablo con él"). Las respuestas fueron puntuadas en una escala tipo Likert de 1 (Totalmente en desacuerdo) a 5 (Totalmente de acuerdo). La consistencia interna fue de 0.91.

\section{Motivación intrínseca}

Se usó el factor Motivación Intrínseca del cuestionario de regulación de conducta en el ejercicio, creado por Wilson, Rogers, Rodgers y Wild (2006) y validado al contexto español por González-Cutre, Sicilia y Fernández (2010). El instrumento, encabezado por la afirmación: "Yo hago entrenamiento de musculación..." estaba compuesto por cuatro ítems (p. ej., "Porque creo que el ejercicio es divertido", "Porque disfruto con las sesiones de ejercicio", "Porque encuentro el ejercicio una actividad agradable" y "Porque me resulta placentero y satisfactorio el hacer ejercicio"). Las respuestas fueron puntuadas en una escala Likert de 0 (Totalmente en desacuerdo) a 4 (Totalmente de acuerdo). La consistencia interna fue de 0.81 .

\section{Disfrute}

Se empleó la Physical Activity Enjoyment Scale, validada al contexto español por Moreno et al. (2008), para medir el disfrute en la práctica de actividad física. La escala, precedida de la afirmación: "Cuando estoy activo/a..." estaba compuesta por 16 ítems que evaluaban el disfrute de forma directa (p. ej., "Disfruto", "Es muy excitante", "Lo encuentro agradable") e inversa (p. ej., "Me aburro", "Me frustra", "No me gusta"). Las respuestas fueron puntuadas en una escala tipo Likert cuyos rangos de puntuación oscilaban entre 1 (Totalmente en desacuerdo) y 5 (Totalmente de acuerdo). La consistencia interna fue de 0.81 .

\section{Compromiso deportivo}

Se empleó la escala denominada Compromiso Deportivo Futuro, elaborada por Orlick (2004) y tomada de Domínguez (2009) para evaluar el compromiso individual del practicante hacia la práctica comprometida y continuada con su deporte. El cuestionario, encabezado por la afirmación: "En mis entrenamientos de musculación..." estaba compuesto por nueve ítems (p. ej., "Realmente quiero ser excelente entrenando musculación”). Las respuestas fueron dadas sobre una escala tipo Likert de 1 (Totalmente en desacuerdo) a 5 (Totalmente de acuerdo). La consistencia interna fue de 0.7.

\section{Procedimiento}

Una vez determinada la muestra objeto de estudio, se estableció contacto con el máximo responsable de los centros deportivos seleccionados y con los monitores de musculación presentes en estos centros para informarles de nuestros objetivos y para pedirles su colaboración. Los cuestionarios se administraron estando presente el investigador principal, para poder hacer una breve explicación del objetivo del estudio, informar a los participantes sobre cómo cumplimentar los 
instrumentos de recogida de datos y solucionar las posibles dudas que pudiesen surgir durante el proceso. Además, se insistió en el anonimato de las respuestas, voluntariedad y en la necesidad de que contestasen con la mayor sinceridad posible. El cuestionario fue rellenado por los participantes en un local tranquilo y sin ruidos, antes de la práctica habitual de la actividad física. El tiempo necesario para completar el total de las escalas fue de aproximadamente 15 minutos, variando esta cifra ligeramente según la edad de los participantes.

\section{Definición de variables}

En el estudio se han evaluado variables conductuales (composición de la comunicación tanto verbal como no verbal) y variables de la Teoría de la Autodeterminación (motivación, disfrute y compromiso) que se ven reflejadas en la Tabla 1.

\section{TABLA 1}

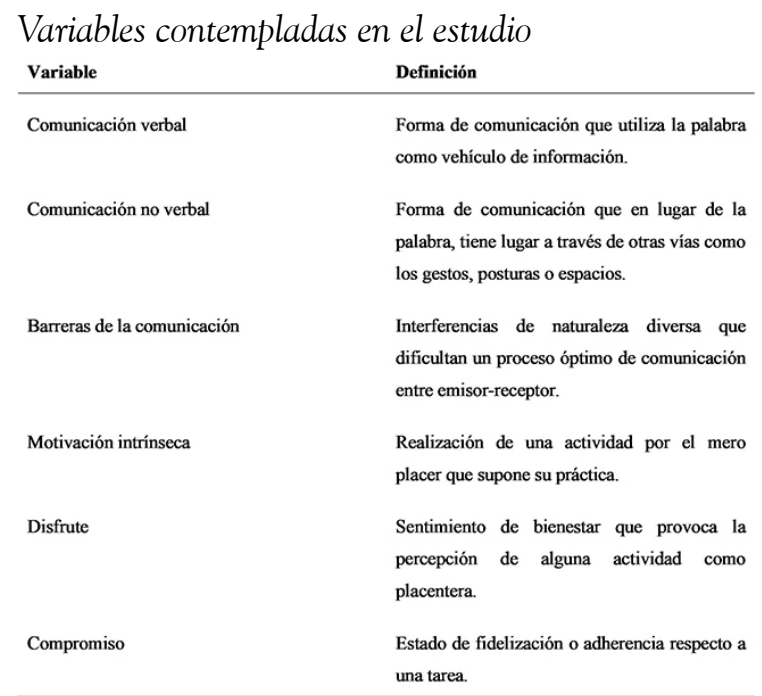

Fuente: elaboración propia.

\section{Análisis de datos}

El estudio realizado es de carácter descriptivo y correlacional. Para saber qué importancia dan los practicantes de musculación a los tipos de comunicación del monitor, la motivación y el disfrute. Se realizó el cálculo de los estadísticos descriptivos de todas las variables objeto de estudio (medias y desviaciones estándar) y luego se investigó cómo se relacionaban dichas variables, para lo cual se llevó a cabo un análisis de correlaciones bivariadas. Para conocer la consistencia interna de cada una de las dimensiones, se utilizó el coeficiente de alfa de Cronbach. Asimismo, se comprobó el poder predictivo de la comunicación sobre el compromiso deportivo futuro del practicante a través de un análisis de regresión lineal múltiple por pasos. El tratamiento estadístico de los datos se efectuó con el paquete estadístico SPSS 21.0.

\section{Resultados}

\section{Análisis descriptivos y de correlación}

La comunicación no verbal: gestos, miradas o realización de gestos técnicos, fue más valorada que la comunicación verbal, forma más concisa, directa, adecuada, clara y respetuosa. La evitación de barreras, es decir, que el monitor no implante barreras de comunicación, como ser estúpido al hablar o evitar que el practicante se comunique, presentó una media de 4.18. La motivación intrínseca, o sea que el practicante esté determinado interiormente por la práctica de musculación, presentó una media de 3.29. El disfrute, que consiste en practicar musculación por mero goce, tuvo una media de 4.4 y la del compromiso deportivo, que es la intención futura de seguir practicando musculación fue de 3.58. En el análisis de correlación, se observó que todos los factores se relacionaban de forma positiva y significativa entre sí (Tabla 2).

\section{TABLA 2}

Estadísticos descriptivos y correlaciones de las variables de estudio

\begin{tabular}{|c|c|c|c|c|c|c|c|c|c|}
\hline \multirow[b]{2}{*}{ Variables } & \\
\hline & $\mathrm{M}$ & $\mathrm{DE}$ & $\alpha$ & 1 & 2 & 3 & 4 & 5 & 6 \\
\hline 1. Comunicación verbal & 3.77 & 0.74 & 0.68 & - & $0.72^{* *}$ & $0.7^{* *}$ & $0.16^{* *}$ & $0.34^{* *}$ & $0.11^{*}$ \\
\hline 2. Comunicación no verbal & 3.78 & 0.81 & 0.69 & - & - & $0.65^{* *}$ & $0.24^{* *}$ & $0.3^{* *}$ & $0.13^{* *}$ \\
\hline 3. Evitación de barreras & 4.18 & 0.69 & 0.91 & - & - & - & $0.18^{* *}$ & $0.27^{* *}$ & $0.11^{*}$ \\
\hline 4. Motivación intrinseca & 3.29 & 0.67 & 0.81 & - & - & - & - & $0.57^{* *}$ & $0.44^{* *}$ \\
\hline 5. Disfrute & 4.40 & 0.43 & 0.81 & - & - & - & - & - & $0.36^{* *}$ \\
\hline 60. Compromiso deportivo futuro & 3.58 & 0.84 & 0.68 & - & - & - & - & - & - \\
\hline
\end{tabular}




\section{Modelo de regresión lineal}

Para comprobar el valor predictivo de la comunicación verbal, comunicación no verbal, evitación de barreras de comunicación, motivación intrínseca y disfrute (variables independientes) de dichos practicantes sobre el compromiso deportivo futuro (variable dependiente), se realizó un análisis de regresión lineal por pasos (Tabla 3). Según el cuarto paso en el análisis de regresión lineal, todas las variables predijeron de forma positiva el compromiso deportivo futuro. La varianza explicada obtenida fue del $20 \%$.

\section{TABLA 3}

Análisis de regresión lineal para la predicción del compromiso futuro

\begin{tabular}{lllll}
\hline & B & SEB & $\beta$ & $\Delta R^{2}$ \\
\hline & 0.8 & 0.47 & & $0.2^{* *}$ \\
Comunicación verbal & 0.11 & 0.09 & $0.11^{*}$ & \\
Comunicación no verbal & 0.24 & 0.18 & $0.11^{*}$ & \\
Evitación de barreras & 0.1 & 0.09 & $0.1^{*}$ & \\
Motivación intrínseca & 0.42 & 0.07 & $0.35^{* *}$ \\
Disfrute & 0.32 & 0.12 & $0.16^{*}$ & \\
\hline
\end{tabular}

Nota. $* \mathrm{p}<0.05 ; *{ }^{*} \mathrm{p}<0.01$.

Fuente: elaboración propia.

\section{Discusión}

Con el propósito de profundizar un poco más el conocimiento de la relación de algunas variables psicosociales del compromiso futuro que los practicantes presentan hacia la práctica de musculación, el objetivo de este estudio fue comprobar la predicción del compromiso en practicantes de musculación a través de las estrategias de comunicación (verbal, no verbal y evitación de barreras) del monitor, la motivación intrínseca y el disfrute en la actividad.

Tal como se hipotetizó, la comunicación eficaz predijo positivamente el compromiso deportivo futuro. Tanto la comunicación verbal, la comunicación no verbal y la evitación de barreras en la comunicación mostraron relaciones positivas y significativas con la motivación intrínseca de los practicantes de musculación. La comunicación no verbal ha sido la más valorada entre los tipos analizados. Este resultado está en la línea de diversas investigaciones en las que se muestra en diferentes ámbitos qué aspectos relacionados con una comunicación adecuada del profesional predicen de manera positiva y significativa la motivación intrínseca de los participantes (Amorose \& Horn, 2000; Standage, Duda, \& Ntoumanis, 2003a, 2003b). Así pues, en otros ámbitos deportivos y en educación física, diferentes autores confirman una relación positiva entre el tipo comunicación que el docente transmite y altos niveles de motivación intrínseca en el alumnado (Amorose \& Smith, 2003; Moreno-Murcia et al., 2013; Nicaise, Cogerino, Bois, \& Amorose, 2006). Sin embargo, al contrario que la presente investigación, no confirmaron el esperado poder predictivo de la comunicación no verbal sobre la motivación intrínseca en estudiantes, pero en la misma línea, Koka y Hein (2005) y Moreno-Murcia et al. (2013) observaron que la comunicación verbal, el conocimiento del rendimiento, la comunicación no verbal y la motivación intrínseca presentaban correlaciones positivas entre sí de manera significativa.

Algunos trabajos en el ámbito de la educación física y el deporte (Liukkonen, Jaakkola, Kokkonen, Laakso, \& Sioni, 2003; Mitchell, 1996; Moreno, Conte, González-Cutre, MartínAlbo, \& Nuñez, 2010; Moreno, González-Cutre, Martín-Albo, \& Cervelló, 2010) indican que la percepción por parte de los estudiantes y deportistas que no haya barrera de comunicación por parte del profesor se relaciona positivamente con la motivación intrínseca, el disfrute de la práctica deportiva y el compromiso deportivo. Además, los resultados de este estudio ponen de manifiesto la importancia de los aspectos no verbales de la comunicación y su posible influencia sobre la motivación de los practicantes de musculación.

El hecho de encontrar una relación positiva y significativa entre los tipos de comunicación verbal y no verbal con la motivación intrínseca enfatiza la importancia de considerar la 
congruencia entre ambas vías de comunicación a la hora de interactuar con el practicante por parte del monitor/entrenador de musculación. De esta manera, los mensajes no verbales que emite el profesional deberían gravitar sobre los mensajes verbales, apoyando y fortaleciendo el significado de estos, mejorando así la comunicación entre el profesional y el practicante de actividad física. Además, el uso de estrategias para evitar las barreras de la comunicación y su relación positiva con la comunicación verbal, no verbal, motivación intrínseca, disfrute y compromiso deportivo futuro, otorgan aún más importancia a la necesidad de dotar al entrenador/monitor de musculación de las estrategias necesarias para mejorar el proceso de comunicación con los deportistas.

Estudios previos en el ámbito físicodeportivo (Matsumoto \& Takenaka, 2004; Moreno, Cervelló, \& González-Cutre, 2007; Vlachopoulos, Karageorghis, \& Terry, 2000) identifican un perfil motivacional que refleja una práctica motivada por la diversión y los beneficios de la actividad, mientras otro perfil muestra una participación caracterizada por la búsqueda de incentivos externos y la ausencia de motivación y de compromiso deportivo. Por lo tanto, conociendo el valor que el practicante otorga al disfrute cuando realiza ejercicio físico, y a la luz de los resultados del presente estudio, sería recomendable que el técnico aplicara estrategias eficaces de comunicación verbal y no verbal, evitando las posibles barreras de comunicación. Este hecho podría provocar mayor diversión y que los practicantes estuvieran más motivados, asegurando así, posiblemente, el compromiso futuro en la actividad.

Las limitaciones de nuestro estudio radican en la naturaleza de la metodología empleada, ya que al ser de tipo correlacional sería necesario emplear una metodología experimental, pues, aunque las variables tengan una relación matemática considerable, no implica necesariamente que haya causa-efecto entre ellas (Quintana, 1989). Los resultados no pueden ser extrapolados más allá de la muestra del estudio. Además, sería necesario ampliar el tamaño de la muestra, implicando a un mayor número de participantes y de centros deportivos o gimnasios. En futuras investigaciones, sería recomendable comprobar estas hipótesis en muestras diferentes y en contextos deportivos diversos, considerando otras variables como el sexo o la práctica deportiva del entorno cercano (Hassandra, Goudas, \& Chroni, 2003), a través de un programa de intervención longitudinal.

\section{Referencias}

Anderson, C. M., \& Martin, M. M. (1995). The effects of communication motives, interaction involvement, and loneliness on satisfaction: A model of small groups. Small Group Research, 26, 118-137.

Arbinaga Ibarzábal, F., \& García García, J. M. (2003). Motivación para el entrenamiento con pesas en gimnasios: un estudio piloto. Revista Internacional de Medicina y Ciencias de la Actividad Física y el Deporte, 3(9), 49-60. Recuperado de http://cdeporte.redir is.es/revista/revista9/artmotivacion.htm

Amorose, A. J., \& Horn, T. S. (2000). Intrinsic motivation: Relationships with collegiate athletes' gender, scholarship status, and perceptions of their coaches' behavior. Journal of Sport and Exercise Psychology, 22, 63-84. http:// dx.doi.org/10.1123/jsep.22.1.63

Amorose, A. J., \& Smith, P. (2003). Feedback as a source of physical competence information: Effects of age, experience and type of feedback. Journal of Sport and Exercise Psychology, 25, 341-359. http:// dx.doi.org/10.1123/jsep.25.3.341

Azorín, F., \& Sánchez-Crespo, J. L. (1986). Métodos y aplicaciones del muestreo. Madrid: Alianza.

Castillo, I., \& Balaguer, I. (2001). Dimensiones de los motivos de práctica deportiva de los adolescentes valencianos escolarizados. Apunts: Educación Física y Deportes, 63, 22-29.

Consejo Superior de Deporte. (2010). Encuesta sobre los hábitos deportivos en España 2010. Madrid: Presidencia del Gobierno. 
Domínguez, M. (2009). Variables motivacionales implicadas en el compromiso deportivo: diseño de un modelo explicativo en el fútbol (Tesis inédita). Universidad de Córdoba, España.

García Ferrando, M. (2006). Posmodernidad y deporte: entre la individualización y la masificación. Encuesta sobre hábitos deportivos de los españoles 2005. Madrid: CSD y CIS.

González-Cutre, D., Sicilia, A., \& Fernández, A. (2010). Hacia una mayor comprensión de la motivación en el ejercicio físico: medición de la regulación integrada en el contexto español. Psicothema, 22 (4), 841-847.

Haerens, L., Aelterman, N., Vansteenkiste, M., Soenens, B., \& Van Petegem, S. (2015). Do perceived autonomysupportive and controlling teaching relate to physical education students' motivational experiences through unique pathways? Distinguishing between the bright and dark side of motivation. Psychology of Sport and Exercise, 16(3), 26-36. http://dx.doi.org/10.1016/ j.psychsport.2014.08.013

Hassandra, M., Goudas, M., \& Chroni, S. (2003). Examining factors associated with intrinsic motivation in physical education: A qualitative approach. Psychology of Sport and Exercise, 4, 211-223. http://dx.doi.org/10.1016/ S1469-0292(02)00006-7

Hernández Mendo, A., \& Garay, P. O. (2003). La comunicación en los contextos deportivos. En A. Hernández Mendo, Psicología del Deporte: Fundamentos 2 (Vol. 1, pp. 29-83). Buenos Aires: Tulio Guterman.

Koka, A., \& Hein, V. (2005). The effect of perceived teacher feed-back on intrinsic motivation in physical education. International Journal of Sport Psychology, 36(2), 91-106.

Liukkonen, J., Jaakkola, T., Kokkonen, J., Laakso, T., \& Soini, M. (2003). Antecedentes de motivación de la actividad física en la juventud finlandesa. Ponencia presentada en el XI Congreso Europeo de Psicología del deporte, Copenhague, Dinamarca.

Matsumoto, H., \& Takenaka, K. (2004). Motivational profiles and stages of exercise behaviorchange. International Journal of Sport and Health Science, 2, 89-96.

Mitchell, S. A. (1996). Relationships between perceived learning environment and intrinsic motivation in middle school physical education. Journal of Teaching in Physical Education, 15, 369-383. http:// dx.doi.org/10.1123/jtpe.15.3.369

Moreno-Murcia, J. A., Huéscar, E., Peco, N., Alarcón, E., \& Cervelló, E. (2013). Relación del feed-back y las barreras de comunicación del docente con la motivación intrínseca de estudiantes adolescentes de educación física. Anales de Psicología, 29(1), 257-263. http:// dx.doi.org/10.6018/analesps.29.1.161881

Moreno-Murcia, J. A., Huéscar, E., Peco, N., Alarcón, E., \& Cervelló, E. (2012). Desarrollo y validación de escalas para la medida de la comunicación en Educación Física y relación con la motivación intrínseca. Universitas Psychologica, 11(3), 957-967.

Moreno, J. A., Cervelló, E., \& González-Cutre, D. (2007). Young athletes' motivational profiles. Journal of Sports Science and Medicine, 6, 172-179.

Moreno, J. A., Cervelló, E. M., Marcos, P. J., \& Martín, E. H. (2010). Importancia de la valoración del comportamiento autónomo del practicante para el disfrute en programas de ejercicio físico acuático. Cuadernos de Psicología del Deporte, 10(1), 57-70.

Moreno, J. A., Conte, L., González-Cutre, D., Martín-Albo, J., \& Nuñez, J. L. (2010). Effects of a task climate intervention on students' motivation in sport instruction. Estudios de Psicología, 31 (1), 67-77.

Moreno, J. A., González-Cutre, D., Martín-Albo, J., \& Cervelló, E. (2010). Motivation and performance in physical education: An experimental test. Journal of Sport Science and Medicine, 9, 79-85. 
Moreno, J. A., González-Cutre, D., Martínez, C., Alonso, N., \& López, M. (2008). Propiedades psicométricas de la Physical Activity Enjoyment Scale (PACES) en el contexto español. Estudios de Psicología, 29(2), 173-180.

Morreale, S. P., Osborn, M. M., \& Pearson, J. C. (2000). Why communication is important: A rationale for the centrality of the study of communication. Journal of the Association for Communication Administration, 29, 1-25.

Muñoz, J. M., Carreras, M. R., \& Braza, P. (2004). Aproximación al estudio de las actitudes $\mathrm{y}$ estrategias de pensamiento social $\mathrm{y}$ su relación con los comportamientos disruptivos en el aula en la educación secundaria. Anales de Psicología, 20(1), 81-91.

Nicaise, V., Cogerino, G., Bois, J., \& Amorose, A. J. (2006). Students' perceptions of teacher feedback and physical competence in physical education classes: Gender effects. Journal of Teaching in Physical Education, 25(1), 36-57. http://dx.doi.org/10.101123/ jtpe.25.1.36

Orlick, T. (2004). Entrenamiento mental: cómo vencer en el deporte y en la vida. Barcelona: Paidotribo.

Poyatos, F. (2003). La comunicación no verbal: algunas de sus perspectivas en estudio e investigación. Revista de Investigación Lingüística, 6(2), 67-83.

Quintana, C. (1989). Elementos de inferencia estadística. Costa Rica: Editorial de la Universidad de Costa Rica.

Reeve, J., Vansteenkiste, M., Assor, A., Ahmad, I., Cheon, S. H., Jang, H.,... Wang, C. K. J. (2014). The beliefs that underlie autonomy-supportive and controlling teaching: A multinational investigation. Motivation and Emotion, 38(1), 93-110. http://dx.doi.org/ 10.1007/ s11031-013-9367-0

Sánchez, D. L., \& Viciana, J. (2002). Análisis del discurso de un entrenador de fútbol. Comparación entre dos situaciones diferentes de competición. Motricidad, 8, 161-174.
Scanlan, T. K., \& Simons, J. P. (1992). The construct of sport enjoyment. En E. G. C. Roberts (Ed.), Motivation in sport and exercise (pp. 199-215). Champaign, IL: Human Kinetics.

Smaby, M. H., Harrison, T. C. \& Nelson, M. (1995). Elementary school counselors as a total quality management consultans. Elementary School Guidance and Counseling, 29, 310-319.

Sousa, C., Torregrosa, M., Viladrich, C., Villamarín, F., \& Cruz, J. (2007). The commintment of young soccer players. Psicothema, 19(2), 256-262.

Standage, M., Duda, J. L., \& Ntoumanis, N. (2003a). A model of contextual motivation in physical education: Using constructs from self-determination and achievement goal theories to predict physical activity intentions. Journal of Educational Psychology, 95(1), 97-110. http:// dx.doi.org/10.1037/0022-0663.95.1.97

Standage, M., Duda, J. L., \& Ntoumanis, N. (2003b). Predicting motivational regulations in physical education: The interplay between dispositional goal orientations, motivational climate and perceived competence. Journal of Sports Sciences, 21, 631-647. http:// dx.doi.org/10.1080/0264041031000101962

Tobón, S. (2004). Formación basada en competencias. Bogotá: Ecoe.

Vlachopoulos, S. P., Karageorghis, C. I., \& Terry, P. C. (2000). Motivation profiles in sport: A self-determination theory perspective. Research Quarterly for Exercise and Sport, 71(4), 387-397.

Wilson, P. M., Rogers, W. T., Rodgers, M., \& Wild, T. C. (2006). The Psychological Need Satisfaction in Exercise Scale. Journal of Sport and Exercise Psychology, 28, 231-251.

\section{Notas}

* Artículo de investigación. 